\title{
UN ACERCAMIENTO A LA IMPLEMENTACIÓN DE POLÍTICAS PÚBLICAS EN COMUNIDADES RURALES EN EL OCCIDENTE DE MÉXICO. ESTUDIO DE CASO EN LA COMUNIDAD "LA CALETA", MUNICIPIO DE CABO CORRIENTES, JALISCO, MÉXICO
}

\section{APPROACH TO THE IMPLEMENTATION OF PUBLIC POLICIES IN RURAL COMMUNITIES IN THE WEST OF MEXICO. CASE STUDY IN THE COMMUNITY OF "CABO CORRIENTES", JALISCO, MEXICO}

\author{
Antonio Saldaña - Salazar ${ }^{1}$ \\ Martha Alicia González - Bueno ${ }^{2}$ \\ Armando Ramírez -Jiménez ${ }^{3}$ \\ 123 Docentes-investigadores de la Universidad Autónoma de Nayarit. México. Integrantes \\ del Cuerpo Académico en Consolidación "Políticas Educativas, Lenguas y Turismo".
}

Recibido: 11.12.17

Aprobado: 24.08 .18

DOI:10.15517/isucr.v20i41.38779

\section{Resumen}

El turismo como una actividad dinámica, que promueve una diversidad de actividades económicas en su entorno, también es capaz de propiciar condiciones adversas al medio ambiente. Por las características de ubicación geográfica, litorales, condiciones hidrográficas, y demás elementos propios del relieve de nuestro país; México puede aprovechar mejor su potencial turístico. Sin embargo, en los últimos años, México ha perdido competitividad frente a destinos de países emergentes en donde se ha privilegiado la inclusión de las comunidades y la conservación de recursos naturales en la oferta turística del presente siglo: desarrollo de turismo comunitario desde el enfoque de la sustentabilidad. Por otra parte, las políticas sociales implementadas por el Estado, no han demostrado efectividad frente a las desgracias económicas que se viven en la mayoría de comunidades rurales (por ejemplo, cambios climáticos drásticos, falta de apoyo al campo, pérdidas de cosechas por plagas, migración hacia las ciudades, entre otras causas).

En este artículo se presenta un primer acercamiento a las políticas públicas empleadas en una comunidad rural a partir de un estudio de caso exploratorio, donde se evidencian algunos de los factores sociales que más impactan a una pequeña comunidad de pescadores, que se localiza en la región del pacifico mexicano, centro occidente del país, llamada playa "Las Caletas". Se recuperan datos obtenidos de un estudio socio-económico aplicado en esta comunidad rural por los autores (Saldaña et al, 2017), con el propósito de analizarlos desde la perspectiva de las políticas públicas empleadas por los diferentes niveles de gobierno. 
La metodología empleada en este estudio documental es de tipo exploratorio e interpretativo, de observación y recolección de testimonios de los residentes de la comunidad quienes son parte del contexto referencial y contextual del presente estudio de caso.

Con el análisis de los datos encontrados y testimonios presentados, se proponen algunas estrategias viables de desarrollo comunitario y medidas de gestión en concordancia con el aprovechamiento y conservación de los recursos naturales de la región, todo ello con el compromiso de los locales para mejorar la sostenibilidad del producto turístico.

Palabras-clave: México; Políticas públicas; Turismo; Jalisco;

\begin{abstract}
:
This article presents a first approach to the public policies used in a rural community from an exploratory case study, where some of the social factors that most impact a small fishing community, located in the region, are evidenced. from the Mexican Pacific, the western center of the country, called "Las Caletas" beach. Data obtained from a socio-economic study applied in this rural community by the authors are recovered (Saldaña et al, 2017), with the purpose of analyzing them from the perspective of the public policies used by the different levels of government.

The methodology used in this documentary study is of an exploratory and interpretative type, of observation and collection of testimonies of the residents of the community who are part of the referential and contextual context of this case study.

With the analysis of the data found and testimonies presented, some viable community development strategies and management measures are proposed in accordance with the use and conservation of the natural resources of the region, all with the commitment of the premises to improve sustainability of the tourist product.
\end{abstract}

Keywords: México; Public policies; Tourism; Jalisco;

\title{
Introducción
}

Este estudio aborda al fenómeno turístico como una actividad dinámica, que promueve una diversidad de actividades económicas en su entorno, con capacidad para propiciar condiciones adversas al medio ambiente.

México, por sus características de ubicación geográfica, litorales, condiciones hidrográficas, y demás elementos propios del relieve con que cuenta, es un país que debe aprovechar mejor su potencial turístico. No obstante, en los últimos años, México ha perdido competitividad frente a destinos de países emergentes en donde se ha privilegiado la inclusión de las comunidades y la conservación de recursos naturales en la oferta turística del presente siglo: desarrollo de turismo comunitario desde el enfoque de la sustentabilidad (SECTUR, 2013). En este punto, es importante señalar qué se entiende por turismo comunitario a efectos de darle mayor claridad y enfoque a las ideas que se exponen en este estudio. En este aporte de ideas no se pretende identificar los principios del turismo comunitario, sino más bien ver al turismo comunitario como una de las actividades de turismo que se desarrolla en el medio rural a través de diferentes servicios turísticos, pero con la especificidad de ser administrado bajo modelos de gestión comunitaria, cuya operación implica la organización de asambleas de indígenas y campesinos quienes tomas decisiones tanto en el manejo y conservación de 
los recursos naturales y patrimonio cultural como en la repartición de las utilidades de la actividad turística.

En este sentido, el turismo comunitario debe asumirse como una concepción de organización social-productiva en comunidades rurales donde se propicie un desarrollo integral que mitigue la pobreza a través de la generación de empleos e ingresos económicos, evitando con ello la migración de su población, fomentando una distribución justa de ingresos, y contribuyendo a la conservación del medio ambiente. En cuanto al concepto de políticas sociales, estas se refieren a las estrategias y políticas concretas que tiene el Estado para construir una sociedad cohesionada y equitativa, con mayor integración social, donde se faciliten tanto los intereses individuales como los de la comunidad. En la temática que nos ocupa, esto constituye una pieza clave para la creación de pequeñas y medianas empresas comunitarias donde se privilegie la preservación del medio ambiente y estilos de vida.

De acuerdo a Kay (2007), el turismo comunitario debe asumirse no como una actividad productivista y mercantil, sino mas bien debe contar con una visión que considere un manejo razonable de los recursos, que sea respetuosa del medio ambiente, y responsable en el entorno social. Asimismo, Orgaz (2013), quien realiza una revisión exhaustiva de la literatura en torno al concepto y noción del turismo comunitario, sostiene que es el tipo de actividad turística realizada por los residentes de la comunidad. Son estos quienes se involucran en la gestión y planificación, administración, y conservación de los recursos naturales, buscando siempre la sostenibilidad de la actividad, cuyos beneficios son utilizados para bien de la comunidad.

El turismo comunitario en América Latina es un fenómeno que data a partir de las últimas tres décadas y se genera en un contexto de grandes cambios económicos, sociales y políticos donde convergen la liberación de flujos comerciales y financieros, la vigencia de los nuevos paradigmas de desarrollo sostenible y la responsabilidad social de la empresa. Maldonado (2007) reconoce que frente al fenómeno de la masificación del turismo, un creciente segmento de turistas reclama productos novedosos, vivencias culturales autenticas y destinos de naturaleza originales. De ahí que las nuevas corrientes del turismo buscan espacios interesantes en contacto con comunidades rurales y el medio ambiente, que conjuguen atributos de originalidad y autenticidad.

En el mismo tenor, el turismo comunitario rural se presenta como una fuente de oportunidades para aquellas comunidades que desean conservar sus raíces, identidad cultural, fomentar el conocimiento de su lengua, formas de vivir, de su ecosistema, y de la cosmovisión del mundo que los rodea. Por lo que resulta una oportunidad para los residentes del lugar para concientizar a los turistas sobre el cuidado al medio ambiente a través del fomento de valores y respecto hacia la naturaleza y demás aspectos relacionados con el patrimonio histórico y cultural de la comunidad.

No obstante lo anterior, el turismo comunitario enfrenta una realidad un tanto cruda, llena de restricciones financieras para el apoyo productivo a la pequeña empresa, discriminaciones y accesibilidad a servicios públicos, infraestructura en comunicaciones, entre otros factores de gestión gubernamental que obstaculizan el desarrollo de actividades de turismo comunitario en México.

Las experiencias vertidas de países como Chile, Perú, Ecuador, Costa Rica y México en torno al desarrollo de turismo comunitario enfocado a la conservación de las especies, tanto de flora y fauna, han aportado diversos elementos de sustentación para la realización de este artículo. 


\section{Desarrollo del estudio}

Hoy en día, especialmente cuando la actividad del turismo en masa o tradicional (turismo de sol y playa) -con respecto a algunos destinos turísticos mexicanos, parece estar desgastada, se hace necesario realizar un análisis reflexivo sobre la pertinencia de los actuales productos turísticos que se han venido desmejorando en los últimos años y que ya no resultan atractivos para las nuevas generaciones.

De acuerdo a las cifras publicadas por el Instituto Nacional de Estadística e Informática (INEGI, 2014), al menos en México, durante el primer trimestre de 2014, algunos destinos como Acapulco, Guerrero, Veracruz, y Michoacán, dependientes del mercado nacional, están resintiendo los efectos de una economía que se mueve lentamente. Esto aunado a problemas sociales de inseguridad, falta de creatividad y competencia en la oferta turística, pero especialmente, una baja notable en la economía de quienes más aportan al sector turístico en función de sus ingresos: la clase media en México.

A este respecto, conviene enfatizar que bajo la existencia de una estructura social debilitada en sus instituciones públicas el potencial turista ha tenido que ajustar sus formas de relajación, descanso o entretenimiento, buscando opciones que sean más seguras y económicamente más accesibles.

De tal suerte que se ha creado un modelo "alternativo" de hacer turismo que está siendo amoldado por las exigencias actuales de tipo social, económico, cultural y político. De ahí que se empieza a mirar el territorio con otros ojos y se comienza a hacer una valoración distinta en términos turísticos, de lo natural y lo rural. En particular, con el objeto de precisar y enfocar el concepto de turismo comunitario y su relación con el medio rural, los autores de este artículo decidimos emplear la siguiente apreciación sobre el turismo rural comunitario, cuyo objetivo es contribuir desde las zonas rurales a un turismo sostenible como herramienta de desarrollo económico-social de una localidad determinada.

De acuerdo al Viceministerio de Turismo de Perú, las características que se destacan en el Turismo Rural Comunitario ${ }^{1}$ que se identifican como un desarrollo sostenible son las siguientes:

- Aquella que se basan en la participación y gestión local

- Aquellas que se desarrollan en el medio rural

- Aquellas que son responsables con el medio ambiente

- Aquel que privilegia el valor de experiencia

- Aquel cuya oferta de productos se basa en la cultura rural

- Aquel que permite la interacción entre el visitante y el anfitrión.

En este primer acercamiento al estudio de las políticas sociales es pertinente destacar que existe interés de la comunidad de la playa "Las Caletas" donde se pretende generar algunas actividades de turismo comunitario, con el objeto de mejorar la situación económica de las familias que ahí radican y, con ello, complementar sus ingresos económicos derivados de la pesca.

La metodología empleada en este estudio documental es de carácter exploratorio e interpretativo, de observación y recolección de algunos testimonios de locales que fueron empleados en el marco referencial y contextual de esta investigación. El tipo de investigación

\footnotetext{
${ }^{1}$ Fuente: Ministerio de Comercio Exterior y Turismo. Viceministerio de Turismo. Perú. Proyecto Turismo Rural 2007-2010.
} 
es mixta, ya que se basa en alguna proporción en datos cuantificables para buscar una explicación de los eventos observados.

Con el análisis de los datos encontrados y testimonios presentados, se proponen algunas estrategias viables de desarrollo comunitario y medidas de gestión social en función al aprovechamiento y conservación de los recursos naturales de la región, teniendo como eje de control directo a la comunidad en la planificación y gestión de las iniciativas, además de constituirse en la propietaria de negocio turístico.

\section{Marco contextual del estudio}

El turismo comunitario como una modalidad turística en la región centro-occidente de México ofrece grandes potencialidades de desarrollo que pueden ser una alternativa al turismo tradicional.

El turismo comunitario permite la conservación del medio ambiente y la participación de la comunidad local en la gestión turística del territorio.

En primer acercamiento se muestran las potencialidades de desarrollo del turismo comunitario en la localidad de "La Caleta", en donde también se analiza la importancia del turismo comunitario a través de un análisis de las características socio-económicas de la región que justifican el modelo de gestión. Por otra parte se analiza el sitio turístico tanto desde una perspectiva eco-turística, así como desde el tradicional destino turístico. Por último, se exponen los resultados de la investigación documental realizada junto con algunos de los testimonios de locales del lugar.

\section{Objetivos del presente estudio.}

I. Analizar la importancia del turismo comunitario como potencial del desarrollo socioeconómico de la localidad "La Caleta", Municipio de Cabo Corrientes, Jalisco.

II. Identificar la tipología de los visitantes que acuden a la localidad de "Las Caletas", Municipio de Cabo Corrientes, Jalisco.

III. Proponer medidas de gestión social, potencial ecológico y recreacional con el objeto de hacer sostenible su uso. 


\section{Ubicación del objeto de estudio}

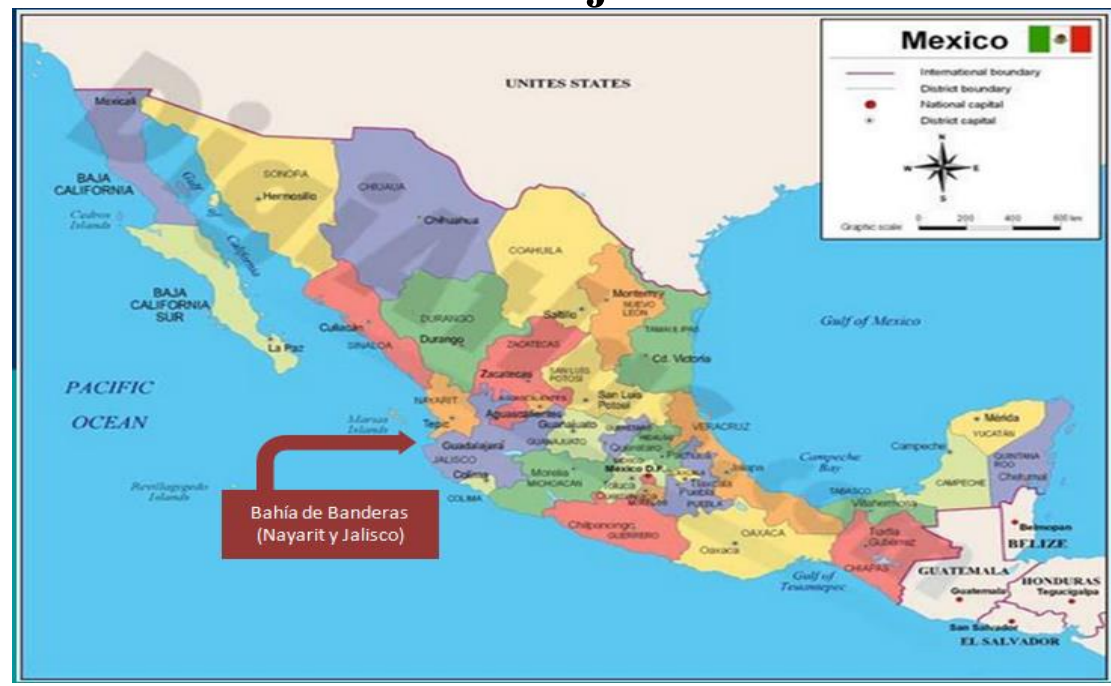

Figura 1. México. Bahía de Banderas localizada entre los Estados de Nayarit y Jalisco.

\section{Micro-localización del objeto de estudio Región Centro-Occidente de México}

La región de Bahía de Banderas se encuentra entre los Estados de Nayarit y Jalisco, en el occidente de México. El estado de Jalisco puede considerarse como la puerta del Pacifico con respecto a la zona centro y noroeste del país. El relieve de Jalisco se caracteriza por el predominio de montañas y la ausencia de llanuras, sobretodo en la región costera.

En la región de la costa norte de Jalisco predominan los climas húmedos tropicales, y es especialmente rica en cuencas fluviales, por ejemplo: las cuencas del Rio Boca de Tomatlán, Rio Cuale, entre otras (ver figura 2).
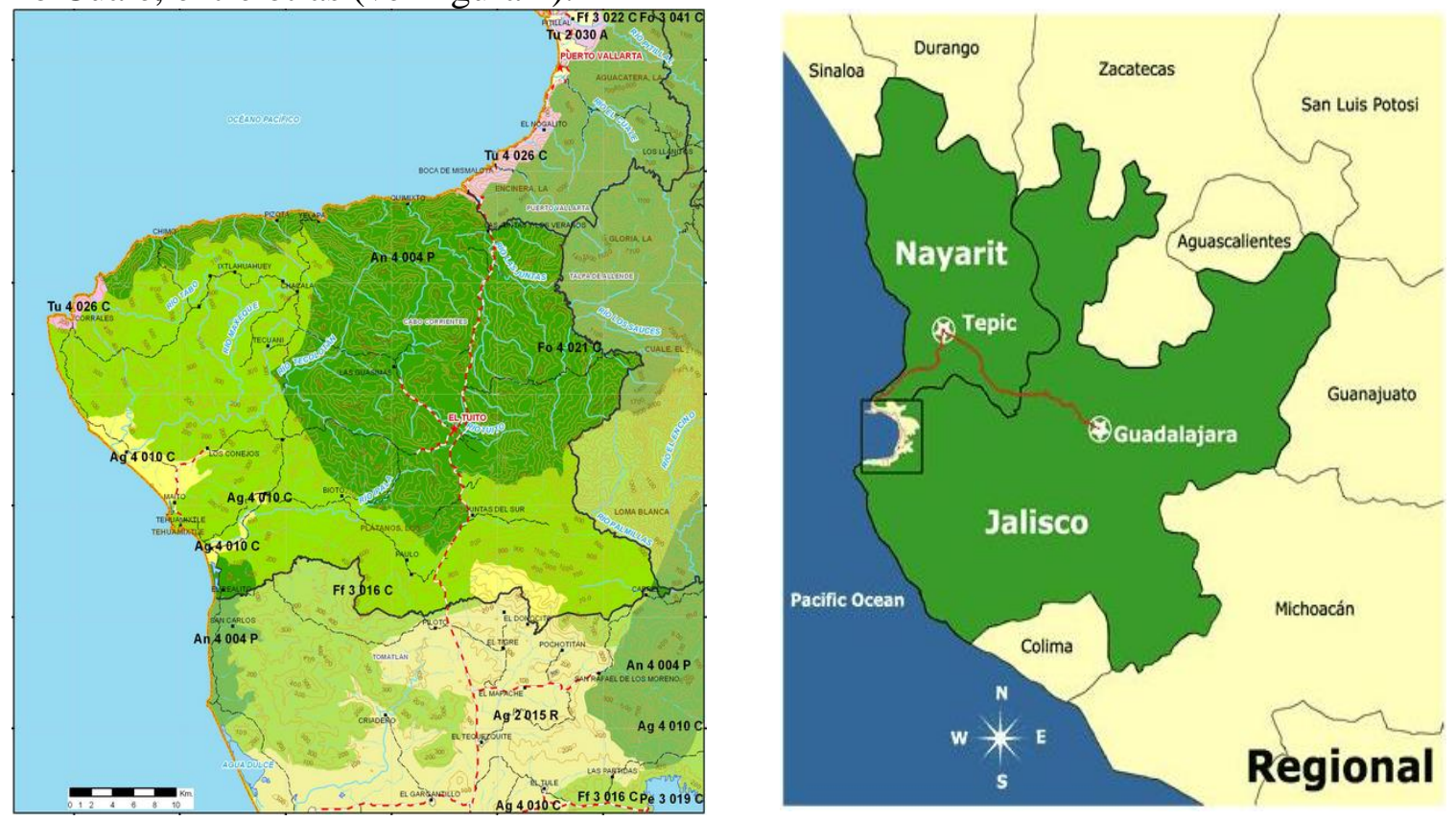
Figura 2. En el mapa de la derecha se muestra la región Centro-Occidente de México, enmarcando en un cuadro la Bahía de Banderas, la cual se ubica justo entre los Estados de

Nayarit y Jalisco localizados en el Pacífico Mexicano. En el mapa de la izquierda se muestra al centro el municipio de Cabo Corrientes, Jalisco. Imágenes tomadas del Gobierno del Estado de Jalisco. (2014). Sección Municipios.

\section{Metodología empleada}

La metodología empleada en el presente estudio se realizó de la siguiente manera.

I. Se llevó a cabo el recuento de visitantes en temporada alta (el conteo se realizó directamente con la empresa que transporta a los visitantes: Vallarta Adventures), atendiendo a las características de procedencia de visitante (extranjero, nacional), nivel de ingresos (alto, medio, bajo), tiempo de pernoctación en el lugar (un día, dos días, tres o más).

II. Entrevista a locales referente a los servicios que ofrece el lugar y lo que buscan los visitantes (identificación de factores de oferta-demanda).

III. Investigación documental basada en los indicadores socio-económicos, servicios públicos y educativos, que realiza el INEGI en el municipio de Cabo Corrientes, Jalisco, donde se localiza la comunidad de estudio.

\section{Resultados}

Indicadores socio-económicos de los pobladores del Municipio de Cabo Corrientes.

La siguiente tabla refleja el nivel de bienestar social y pobreza multidimensional que presentan los pobladores del Municipio de Cabo Corrientes en el Censo de Población y Vivienda realizado en 2010.

\begin{tabular}{|c|c|c|}
\hline \multicolumn{3}{|c|}{ Cabo Corrientes, 2010} \\
\hline Indicadores de incidencia & Porcentaje & Personas \\
\hline \multicolumn{3}{|l|}{ Pobreza multidimensional } \\
\hline Población en situación de pobreza multidimensional & 63.1 & 6,215 \\
\hline Población en situación de pobreza multidimensional moderada & 50.8 & 5,001 \\
\hline Población en situación de pobreza multidimensional extrema & 12.3 & 1,214 \\
\hline Población vulnerable por carencias sociales & 30.0 & 2,957 \\
\hline Población vulnerable por ingresos & 2.5 & 251 \\
\hline Población no pobre multidimensional y no vulnerable & 4.4 & 430 \\
\hline \multicolumn{3}{|l|}{ Privación social } \\
\hline Población con al menos una carencia social & 93.1 & 9,173 \\
\hline Población con al menos tres carencias sociales & 34.5 & 3,398 \\
\hline \multicolumn{3}{|l|}{ Indicadores de carencias sociales } \\
\hline Rezago educativo & 31.6 & 3,118 \\
\hline Acceso a los servicios de salud & 19.4 & 1,915 \\
\hline Acceso a la seguridad social & 76.2 & 7,503 \\
\hline Calidad y espacios de la vivienda & 13.8 & 1,360 \\
\hline Acceso a los servicios básicos en la vivienda & 28.4 & 2,796 \\
\hline Acceso a la alimentación & 41.8 & 4,115 \\
\hline \multicolumn{3}{|l|}{ Bienestar } \\
\hline Población con un ingreso inferior a la linea de bienestar minimo & 28.1 & 2,767 \\
\hline Población con un ingreso inferior a la linea de bienestar & 65.6 & 6,466 \\
\hline
\end{tabular}

FUENTE: IIEG, Instituto de Información Estadistica y Geográfica del Estado de Jalisco con base en, estimaciones del CONEVAL con base en INEGI, MCS-ENIGH 2010 y la muestra del Censo de Población y Vivienda

${ }^{1}$ Se reporta el porcentaje de la población con cada carencia social.

Figura 3. Pobreza multidimensional e indicadores de incidencia. Fuente: IINEG (2010). Instituto de Información Estadística y Geografía del Estado de Jalisco.

Como se muestra en la figura 3, más de la mitad de la población del municipio se encuentra entre pobreza moderada y extrema, lo cual refleja un alto grado de seguridad social (fuentes 
de empleo, infraestructura, apoyo de programas sociales). Por otra parte, existe un alto rezago educativo (31.6\%), lo cual hace más vulnerable la situación económica y social del municipio.

\section{Tipología de visitantes que visitan la playa "Las Caletas"}

Enseguida se muestra el tipo de turistas que acuden a este sitio. La clasificación de este análisis se llevó a cabo en función a: procedencia, profesión, y tiempo de pernoctación en el lugar (playa "Las Caletas"). La muestra estuvo compuesta por 100 turistas elegidos al azar en temporada alta (entre los meses de Julio y agosto de 2014)

Tabla 1: Procedencia de visitantes extranjeros y nacionales.

\begin{tabular}{|l|l|l|l|}
\hline \multicolumn{2}{|l|}{$\begin{array}{l}\text { Procedencia de visitantes extranjeros } \\
(\mathbf{1 0 0})\end{array}$} & $\begin{array}{l}\text { Estados de México identificados por } \\
\text { Región (100\%) }\end{array}$ & Porcentaje \\
\hline País o región & Porcentaje & Región de & $39 \%$ \\
\hline EEUU & $16 \%$ & $\begin{array}{l}\text { Occidente } \\
\text { México }\end{array}$ & $24 \%$ \\
\hline Canadá & $9 \%$ & Centro de México & $9 \%$ \\
\hline Europa & $7 \%$ & Sur de México & $19 \%$ \\
\hline Asia & $6 \%$ & Norte de México & $7 \%$ \\
\hline $\begin{array}{l}\text { América Central y } \\
\text { América del Sur }\end{array}$ & $4 \%$ & Noreste de México & $7 \%$ \\
\hline México & $58 \%$ & Sureste de México & $2 \%$ \\
\hline
\end{tabular}

En tabla 1 se muestra la información recuperada en la empresa privada (Vallarta Adventures) que es concesionaria del uso de la infraestructura existente en "Las Caletas". En este reporte se observa que predominan los turistas nacionales en temporada de verano en relación con el turismo extranjero, específicamente durante los meses de Julio y Agosto. Se muestra además un flujo de visitantes importante que proviene de EEUU y Canadá en contraste con turismo latinoamericano.

En cuanto a las regiones de México, en la región occidente se observa el mayor flujo de turistas que provienen de estados colindantes, lo que presupone la ventaja de la ubicación geográfica del destino, menor costo en el transporte de visitantes (ya sea por tierra o por aire). El segundo punto de procedencia (centro del país) en cuanto el porcentaje detectado (24\%) muestra la oferta de vuelos, costo, y frecuencia de vuelos al destino (Puerto Vallarta) y, por supuesto, refleja la demanda de los turistas en función a la elección del destino sol y playa. 
Tabla 2: Nivel de ingresos identificado por visitante.

\begin{tabular}{|c|c|c|c|}
\hline \multicolumn{4}{|l|}{ Nivel de ingresos } \\
\hline Tipo de turismo & $\begin{array}{l}\text { Bajo (1-3 salarios } \\
\text { mínimos) }\end{array}$ & $\begin{array}{l}\text { Medio (4-6 salarios } \\
\text { mínimos) }\end{array}$ & $\begin{array}{l}\text { Alto } \quad\left(\begin{array}{lll}7 & \text { o más } \\
\text { salarios mínimos) }\end{array}\right.\end{array}$ \\
\hline Extranjero & $8 \%$ & $56 \%$ & $36 \%$ \\
\hline Nacional & $2 \%$ & $73 \%$ & $25 \%$ \\
\hline
\end{tabular}

En el rubro del nivel de ingresos por visitante se observa un claro despegue en ambos tipos de turismo (nacional y extranjero). Esto significa un mayor aporte al sector turismo de parte de familias de clase media, en contraste con el turismo de altos ingresos. Respecto al turismo de ingresos bajos, este no es significativo en cuanto al aporte de ingresos al sector turismo que visita esta localidad (Las Caletas).

En relación al número de días que los visitantes pernoctan en la localidad, en ninguno de los segmentos de visitantes entrevistados (nacionales y extranjeros) se reportó una duración mayor a un día. Esto posiblemente explica la ausencia de infraestructura adecuada para pernoctar más de un día.

Con base en los testimonios recuperados en la localidad (Las Caletas), los pobladores reportan salarios ajustados a jornadas de ocho horas y prestaciones mínimas de ley. Algunos de ellos reportan que complementan su salario con reparto equitativo de propinas.

En cuanto a los apoyos federales que el gobierno otorga, de acuerdo con los testimonios recabados por los locales, estos han sido muy escasos y realmente no les permiten mejorar su situación económica (por ejemplo, Cruzada Nacional del Hambre).

En los testimonios recogidos se percibe que existe desinterés por parte de las autoridades municipales en atender las necesidades prioritarias, tales como salud, educación, vivienda, infraestructura en comunicaciones, entre otros rubros esenciales.

Se observó que los espacios naturales se encuentran desprotegidos de la depredación del hombre. No hay concientización sobre el cuidado de sus alrededores. Solo existe señalamiento en el punto de desembarque y en un radio de alrededor de 500 metros. Fuera de ese perímetro no existe infraestructura turística de ningún tipo.

El tipo de infraestructura visible (comedores, baños, hamacas, tiendas de recuerdos, pequeño muelle) no permite pernoctar en el lugar por más de un día. Además, no existe un circuito o recorrido turístico que incentive al turista quedarse ahí por más de 5 o 6 horas.

\section{Discusión}

En términos generales, después de analizar los datos recogidos en este estudio se percibe un alto nivel de marginación social en la mayoría de las comunidades rurales del Municipio de Cabo Corrientes, Jalisco, incluyendo la comunidad de estudio. Existen condiciones desfavorables de infraestructura y atención a los servicios públicos más elementales de la población en el lugar. Por lo que se prevé un escenario de mayor reto en la creación de actividades de turismo comunitario y rural, pero a la vez se visualiza un campo virgen en la conservación de las riquezas naturales y culturales con que cuenta el lugar. 
En este sentido, existe una justificación plena para la creación de fuentes alternas de ingresos económicos que las comunidades, especialmente, aquellas que colindan con el litoral costero del pacifico, generen a partir del cuidado, manejo y preservación de los recursos naturales existentes. Si bien es cierto, el ecosistema circundante es prolífico y diverso, este debe ser preservado para futuras generaciones (Saldaña et al, 2017).

Para hacer frente a este reto, el primer imperativo consiste en alentar procesos asociativos que procuren eficientemente la oferta de servicios, con base en un esquema de ordenamiento del espacio comunitario, pero sin desatender el uso sostenible del patrimonio comunitario. En el mismo orden de ideas, Maldonado (2007) hace alusión a una agenda de puntos que los gobiernos locales deberán concretar en conjunto con aquellas organizaciones comunitarias a fin de lograr un marco normativo con políticas adecuadas para su desarrollo, a saber:

1. Definir los roles de los actores locales en el desarrollo del turismo: municipios, organizaciones comunitarias y sociales, universidades, y empresa privada;

2. Promover leyes y normas que regulen e incentiven el ejercicio del turismo comunitario;

3. Concertar políticas y programas, incorporando los objetivos y aportes específicos de las comunidades rurales en los planes estratégicos de desarrollo municipal;

4. Reconocer, proteger y promover las expresiones del patrimonio local, la identidad cultural, sus valores, instituciones y prácticas sociales.

5. Respetar las decisiones de las comunidades y los pueblos indígenas en lo que atañe al uso y destino de sus territorios, incluyendo el derecho a no optar por la actividad turística.

Evidentemente, existen condicionantes para el logro de estas aspiraciones, las cuales estriban en que la autoridad municipal verdaderamente se interese por promover, financiar, y otorgar asesoría técnica especializada para la creación de fuentes de trabajo basadas en un turismo comunitario. En este punto, la comunidad de playa "Las Caletas" no cuenta con el apoyo necesario de parte de las autoridades municipales, ni tampoco cuenta con algún tipo de incentivo tributario, económico que estimule el despegue de esta iniciativa.

No obstante, en la comunidad y sus alrededores se observa un alto potencial de desarrollo turístico susceptible de ser aprovechado para fines del turismo comunitario y rural, y con ello, detonar el desarrollo socio-económico de la región.

Es importante destacar aquí que hoy en día, el gobierno mexicano reconoce en el Plan Nacional de Turismo la sustentabilidad como un concepto base para la implementación de estrategias de desarrollo a efectos de promover la sustentabilidad y desarrollo rural, lo cual, en términos institucionales, da mayor viabilidad a los proyectos de turismo alternativo. Lo anterior tiene fundamento en las nuevas tendencias del mercado turístico internacional que día con día busca nuevas opciones de hacer turismo (nuevos destinos) y al mismo tiempo, busca una diversificación en la oferta de productos turísticos (SECTUR, 2013).

Por otro lado, el gobierno mexicano reconoce que el modelo de turismo convencional (sol y playa) tiene señales de agotamiento. Por lo que se vislumbran áreas de oportunidad para desarrollar productos turísticos atractivos y sustentables en el campo del turismo cultural, de negocios, ecoturismo, de aventura, turismo deportivo, entre otros.

El esquema de desarrollo de proyectos de turismo comunitario y rural que se presenta en la siguiente página (ver figura 4) tiene el fin de ilustrar más sobre la viabilidad de este tipo de proyectos. A partir de las directrices que se presentan en el esquema, se derivan una serie de acciones encaminadas a cumplir con las metas planteadas. Algunas de ellas deberán estar enfocadas a la capacitación integral y permanente de recursos humanos, mientras que otras 
deberán orientarse hacia la búsqueda de organismos, organismos no gubernamentales (ONGs), universidades, $\mathrm{u}$ otras entidades privadas que apoyan y fomentan este tipo de actividades comunitarias en salvaguarda del patrimonio cultural de los pueblos.

Maldonado (2007) sostiene que una vez iniciada la tarea sobre el mejor uso de los recursos naturales y conservación del patrimonio cultural, las comunidades, el gobierno y las organizaciones de la sociedad civil, están obligadas a encontrar y difundir las tecnologías y prácticas de preservación de los ecosistemas y la biodiversidad sustentadas en los conocimientos ancestrales, el uso de energías renovables y no contaminantes, la racionalización del agua, la gestión de desechos sólidos y aguas residuales de origen domestico o turístico.

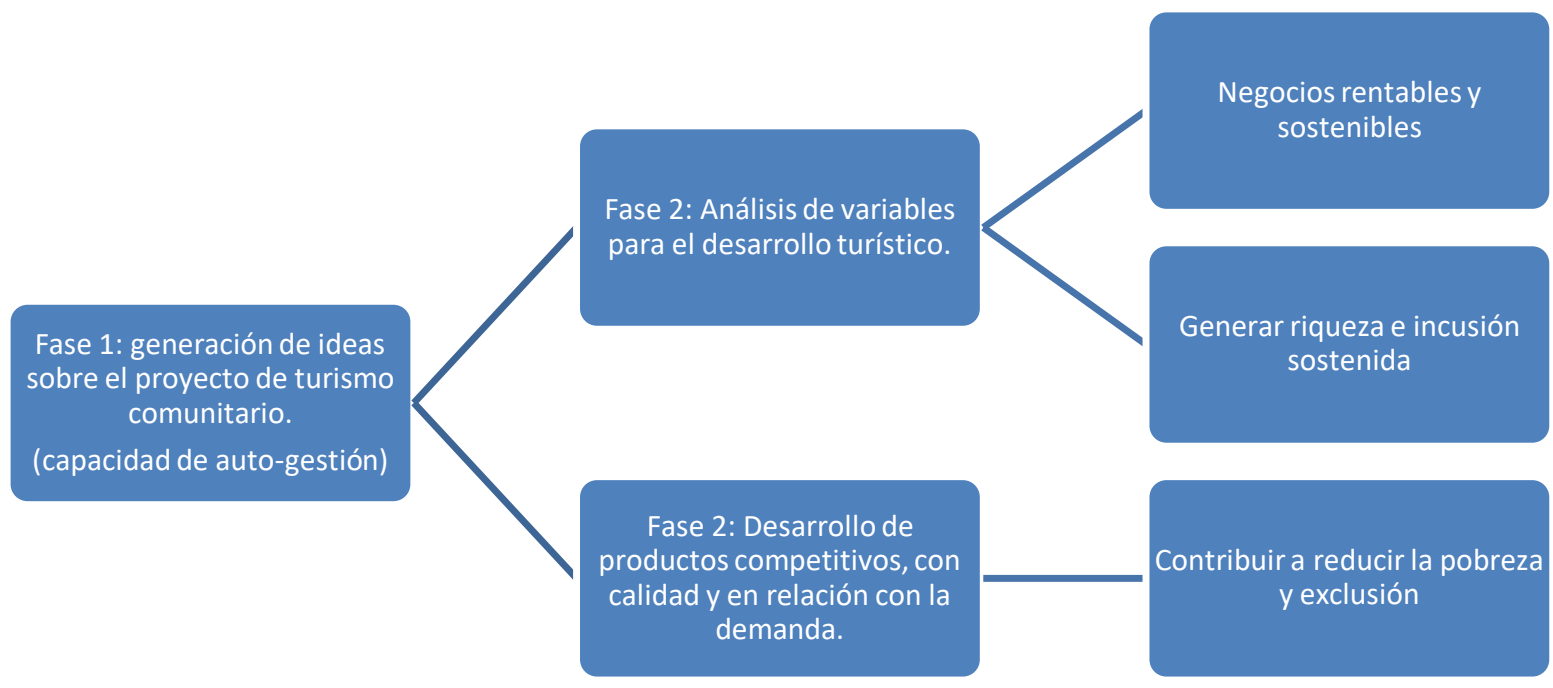

Figura 4. Modelo adaptado del Enfoque propuesto por el Ministerio de Comercio Exterior y de Turismo de Perú (2012). Del mapa de la pobreza al mapa de oportunidades.

\section{Conclusiones}

De acuerdo a estudios socio-económicos recientes, al parecer las estrategias de desarrollo estatal promovidas por el gobierno no han sido tan exitosas como se anuncian. Existe una polarización muy marcada especialmente el sector terciario, por ello, afirman los expertos, es necesario revisar las estrategias de desarrollo social estatal que permitan determinar el impacto en las carencias sociales asociadas a la pobreza de la población (Barrón y Orozco, 2014).

La situación de marginación y pobreza no es menor en los municipios de Puerto Vallarta y Cabo Corrientes, ya que el nivel de marginación y acceso a servicios públicos es también limitado dado al alto grado de migración a este destino turístico.

Figueroa y otros (2012) señalan que unos de los problemas mayores en países subdesarrollados con bajo o nulo crecimiento de la economía es la migración, pobreza y desempleo. En una investigación documental llevada a cabo por Figueroa y otros identifican algunos de los problemas que ocasionan dichas variables en la economía de México. En 
resumen, los autores concluyen aseverando que las políticas públicas no han funcionado, trayendo consigo un rezago social de pobreza, marginación social, desempleo abrupto y altos niveles de migración hacia centros urbanos sobrepoblados en el país, o bien hacia el país vecino del norte: EEUU. La política social ha fallado en sus estrategias y por el contrario ha aumentado la pobreza. Hoy en día hay más pobres que nunca.

En el caso que nos ocupa, la comunidad de playa "Las Caletas", la situación socio-económica de los pobladores no dista mucho de la realidad descrita en el párrafo anterior. Por ello, es necesario que los pobladores de la comunidad tengan la orientación y apoyo de gestión para el manejo de recursos naturales y administración de pequeñas empresas donde ellos puedan intervenir y ser actores en la toma de decisiones. Al mismo tiempo, es importante que cuenten con la asesoría continua de especialistas en el uso adecuado de espacios naturales con fines recreativos.

Por otra parte es necesario fortalecer las políticas sociales (concebidas estas como estrategias del gobierno) que se emplean para atender las necesidades básicas de las comunidades. Evidentemente, las políticas sociales derivadas de las políticas públicas se han caracterizado por destinar altos montos presupuestarios con bajos índices de efectividad real aplicada a las clases más necesitadas. Esta situación, por supuesto contribuye a elevar los índices de pobreza en comunidades rurales con escenarios similares a los que se viven en la comunidad de "La Caleta".

Es entonces imprescindible sistematizar experiencias y proponer estrategias que constituyan las bases para que un futuro, no muy lejano, el turismo comunitario y sustentable sea una estrategia más de desarrollo local para comunidades que requieren de la generación de sus propios medios económicos para subsistir, pero al mismo tiempo, conservar su cultura, tradiciones, y medio ambiente. En este sentido existen evidencias de algunas comunidades han intentado la auto-regulación de recursos económicos y manejo adecuado de eco-sistemas con el apoyo y asesoría de expertos, lo cual ha resultado exitoso.

Algunos casos de lo anterior se encuentran en comunidades rurales de países de América del Sur (Ecuador, Brasil, Chile, Perú y Costa Rica) que han demostrado a través de su buena administración, organización comunal, conservación de las especies y flora, entre otros aspectos, dado que ha sido un esfuerzo constante de parte de la comunidad y los gobiernos locales.

En tal sentido, es necesario tener en cuenta los siguientes puntos que deben ser considerados para el arranque del proyecto de turismo comunitario, con alto grado de inclusión social.

1. Ordenamiento del espacio comunitario;

2. Análisis situacional para determinar la viabilidad del proyecto;

3. Priorización del mercado potencial (análisis de la demanda del servicio);

4. Capacitación de recursos humanos;

5. Innovación, mejora y calidad de los servicios turísticos;

6. Promoción y comercialización del producto turístico.

A manera de conclusión adoptamos la siguiente idea expresada por los especialistas en Organización Internacional del Trabajo (OIT, 2009: 20):

"Mientras que algunos entienden a la responsabilidad social de la empresa como un ejercicio de relaciones públicas o simplemente ser caritativo con los pobres, una industria del turismo genuinamente incluyente se compromete mucho mas. Las diferencias entre una industria del turismo que se basa en la caridad y una que está comprometida con la inclusión son los resultados: solo una industria incluyente puede alcanzar mayores beneficios al mismo tiempo que aporta dignidad humana". 


\section{Referencias bibliográficas}

Barrón Arreola, K. y Orozco Espinosa, P. (2013). Un acercamiento al estudio de la pobreza en Nayarit. Revista de Divulgación Científica FUENTE Nueva Época. Año 5, No. 16. UAN. México.

COEPO (2014). Consejo Estatal de Población. Documento consultado el 2 de Octubre de 2014. En: http://jalisco.gob.mx/coepo Información concentrada del IINEGI.

Figueroa Hernández, E., Ramírez Abarca, O., González Elías, J. M., Pérez Soto, F. \& Espinosa Torres, L. E. (2012). ANÁLISIS DEL DESEMPLEO, LA MIGRACIÓN Y LA POBREZA EN MÉXICO. Revista Mexicana de Agronegocios, XVI(30) 835847. Recuperado en http://www.redalyc.org/articulo.oa?id=14123097006 Consultado el 30 de Enero de 2015.

Gobierno del Estado de Jalisco. (2014). Municipio de Cabo Corrientes, Jalisco. Información consultada el 2 de Octubre de 2014. En: http://www.jalisco.gob.mx/es/jalisco/municipios/cabo-corrientes

INEGI (2014). Instituto Nacional de Estadística y Geografía. Disponible en: http://www.inegi.org.mx/est/contenidos/proyectos/cn/itat/default.aspx Consultada el 2 de Febrero de 2015.

Kay, C. y Breton, V. (2007). La cuestión agraria y los límites del neoliberalismo en América Latina. Iconos. Revista de Ciencias Sociales, num. 28, Quito, Mayo, 2007, pp. 119133. Facultad Latinoamericana de Ciencias Sociales.-Sede Académica de Ecuador.

Maldonado, C. (2007). Fortaleciendo redes de turismo comunitario. Revista REDTURS en América Latina 2007, num. 4, pp. 8-14.

Ministerio de Comercio Exterior y Turismo de Perú. (2012). Viceministerio de Turismo. Programa Nacional de Turismo Rural Comunitario. Documento recuperado en: www.turismoruralperu.gob.pe Consultado el 30 de Enero de 2015.

OIT, Bolwell, D., Weinz, W. (2009). Reducir la pobreza a través del turismo. Oficina del Trabajo. Ginebra, Suiza.

Orgaz, F. (2013). El turismo comunitario como herramienta para el desarrollo sostenible de destinos subdesarrollados. Nómadas.

Saldaña, A., González, M., Ramírez, A., Flores, D. (2017). Perspectiva del Turismo Comunitario. Un camino a la inclusión social de las comunidades y la conservación de los recursos naturales. Estudio de caso. Revista KIKAME. Vol. 3. No. 3. Pags. 93 105. México.

SECTUR (2013). Programa Sectorial de Turismo 2013-2018. Diario Oficial. Tercera Sección. México. 